\title{
DEMONSTRAÇÃO DO CÁlCULO DA LINHA NEUTRA EM VIGAS SUBMETIDAS À FLEXÃO
}

\section{ARTIGO ORIGINAL}

MOURA, Tiago Rodrigues Coelho de ${ }^{1}$

MOURA, Tiago Rodrigues Coelho de. Demonstração do cálculo da linha neutra em vigas submetidas à flexão. Revista Científica Multidisciplinar Núcleo do Conhecimento. Ano 04, Ed. 10, Vol. 10, pp. 99-115. Outubro de 2019. ISSN: 24480959, Link de acesso: https://www.nucleodoconhecimento.com.br/engenhariacivil/calculo-da-linha

\section{RESUMO}

Um dos sistemas construtivos mais usado no Brasil é o concreto armado. Normalmente, as estruturas são constituídas por lajes, vigas, pilares e fundação. Todos esses elementos devem ser dimensionados e verificados a fim de resistir todos os esforços solicitantes. No dimensionamento, especificamente nas vigas e lajes, são feitas várias verificações. Uma das verificações, exigida pela norma vigente, é a posição da linha neutra. Essa linha neutra separa a região comprimida da região tracionada de um elemento submetido à flexão. Este estudo apresentou, de forma didática, o cálculo dessa linha neutra e as equações deduzidas para facilitar o trabalho dos profissionais de engenharia civil. Ao longo do texto, é exposto o passo a passo do cálculo para vigas com diversos tipos de seções transversais: retangular, I, T e trapezoidal. Todos os cálculos aplicáveis a vigas de concreto armado. Cada tipo de seção transversal resultou em equações diferentes. Normalmente, chega-se a

1 Pós-graduado em Gestão de Obras na Construção Civil pela Faculdade Cidade Verde (FCV), Engenheiro Civil pelo Centro Universitário UNIEURO e técnico em Telecomunicações Aeronáuticas pela Escola de Especialista de Aeronáutica (EEAR). 
equações do segundo grau para seções geometricamente retangulares e do terceiro grau para seções com partes triangulares.

Palavras-Chave: linha neutra, concreto, estruturas, flexão, vigas.

\section{INTRODUÇÃO}

Uma análise bem detalhada dos elementos estruturais é de grande importância para garantir a segurança das edificações. As normas da Associação Brasileira de Normas Técnicas (ABNT) amparam, de forma ampla, o dimensionamento de estruturas na construção civil, em especial, a NRB 6118 (ABNT, 2014) que trata das estruturas de concreto armado. Nessa norma estão previstas várias verificações, uma delas é a posição da linha neutra em vigas. A linha neutra é compreendida como um plano que separa duas regiões - a comprimida e a tracionada - num mesmo elemento quando submetido à flexão. Além das verificações especificadas nessa norma, a posição da linha neutra, em relação à fibra mais comprimida, é necessária para calcular a área de aço de tração da viga. Esse breve estudo tem a finalidade de demonstrar o desenvolvimento das equações desse parâmetro, nos elementos estruturais submetidos à flexão simples.

\section{DESENVOLVIMENTO}

\subsection{LINHA NEUTRA EM VIGA DE SEÇÃO TRANSVERSAL QUALQUER.}

A linha neutra, em um elemento sob flexão simples, separa a região comprimida da região tracionada. A Figura 1 exemplifica essas regiões em uma viga com carregamento uniforme. Nesse caso, todas as cargas transversais produzem um momento fletor que determina a posição dessa linha. 
Figura 1 - Linha neutra em viga.

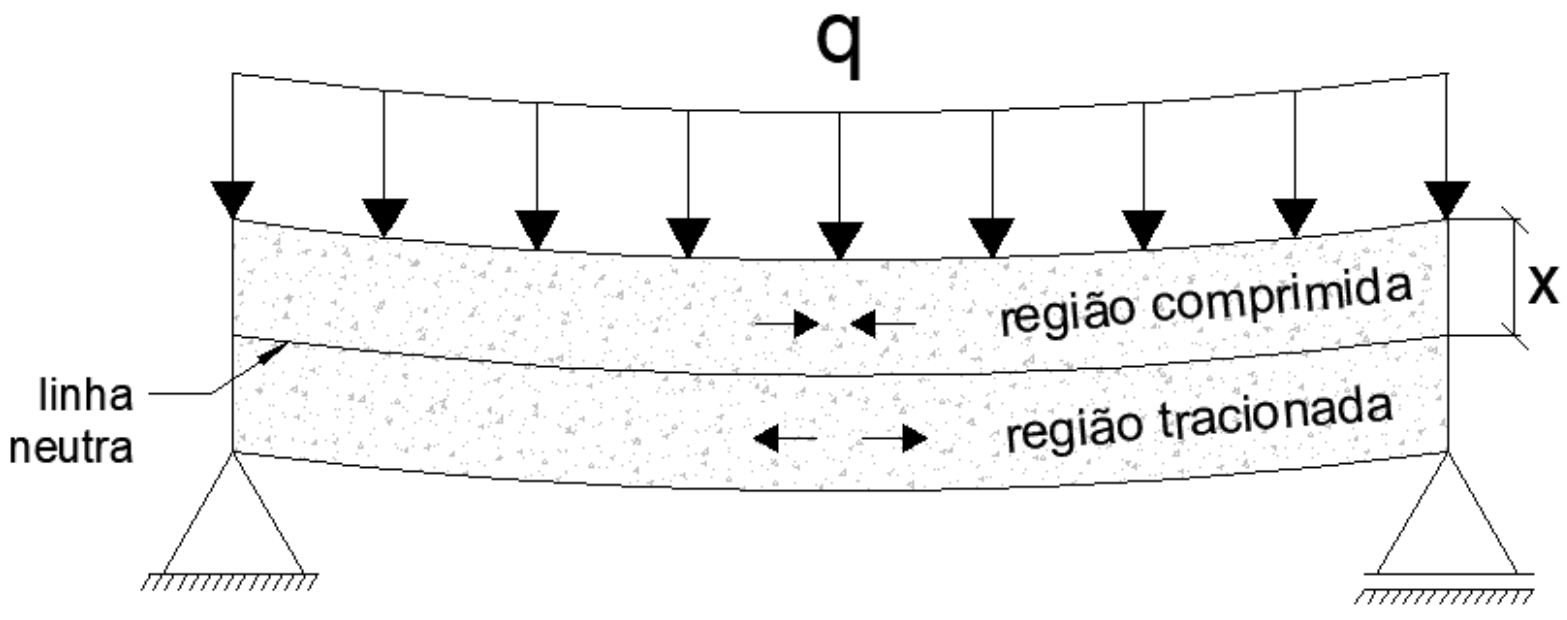

Fonte: própria

Nesses elementos, linha neutra é obtida em função do momento fletor na seção transversal. Fazendo o equilíbrio desse momento, em relação à região comprimida $\mathrm{e}$ ao aço tracionado, obtém-se a equação inicial do problema. Algumas observações previstas na NBR 6118 (ABNT, 2014) devem ser consideradas; a tensão efetiva na compressão do concreto é uma delas.

A tensão de pico do concreto, em acordo com o item 8.2.10 da NBR 6118 (ABNT, 2014), é $85 \%$ da tensão de cálculo. Essa consideração normativa visa garantir a segurança da região comprimida do elemento.

A posição de cálculo da linha é considerada em $80 \%$ da real. Esse modelo de cálculo é adotado para garantir, de forma homogênea, uma distribuição da tensão de compressão, conforme apresentado por Carvalho e Filho (2007). O esquema para demonstração dessa consideração está exposto na Figura 2, onde o gráfico de tensão real do concreto é substituído por outro equivalente, porém, de forma retangular. 
Figura 2 - Linha neutra numa seção qualquer.

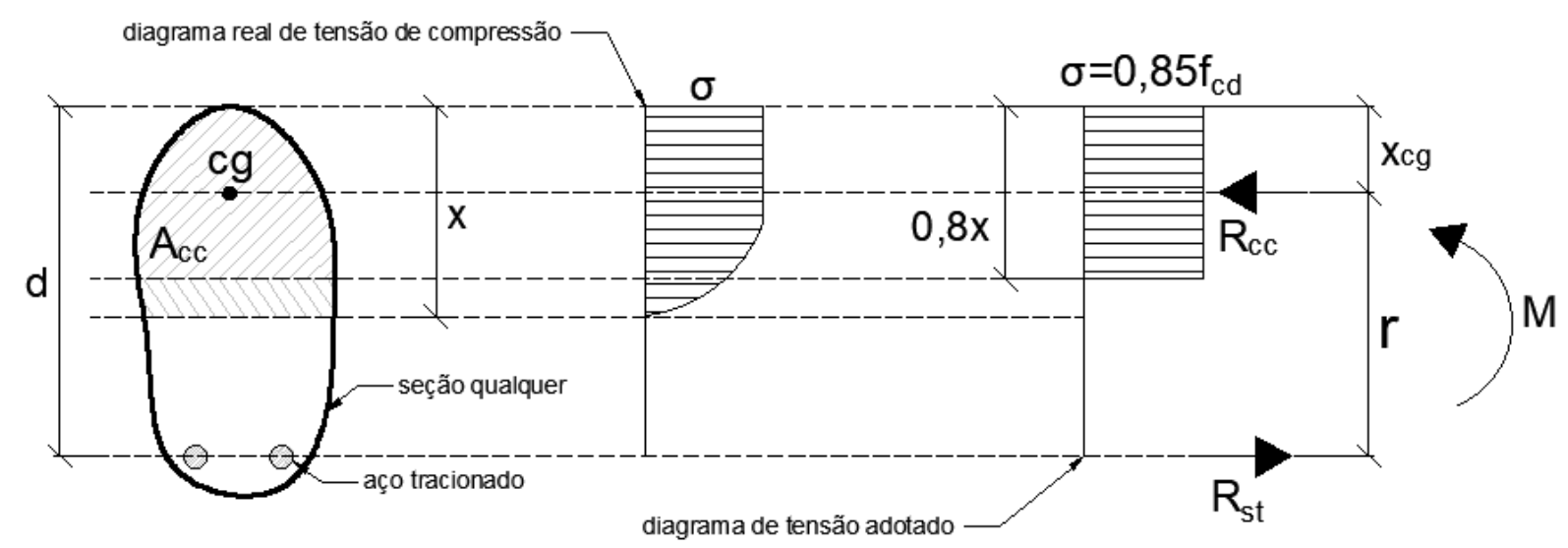

Fonte: própria

Os cálculos são baseados no equilíbrio de momento fletor da região comprimida do concreto e da tração do aço na seção transversal da viga. Conforme a Figura 2, podemos calcular a força na região comprimida $\left(R_{c c}\right)$ em função da tensão e área.

$$
\text { Tensão }=\frac{\text { Força }}{\text { Área }} \rightarrow R_{c c}=0,85 f_{c d} \cdot A_{c c}
$$

Onde

$R_{c c}:$ força de reação da região comprimida;

$f_{c c:}$ tensão de resistência de cálculo do concreto;

$A_{c c}$ área de concreto sob compressão.

A força de reação da região comprimida é concentrada no centro de gravidade da área de concreto sob compressão. A distância $\left(x_{c g}\right)$ desse centro de gravidade até a face mais comprimida da viga pode ser calculado com as seguintes equações:

- Para um contorno da seção transversal definido por uma função $f(x)$ num intervalo de " $a$ " até " $b$ ". 
$x_{c g}=\frac{\int_{a}^{b} f(x) x d x}{\int_{a}^{b} f(x) d x}$

- Para uma seção transversal definida por figuras geometricamente conhecidas.

$$
x_{c g}=\frac{\sum A_{i} x_{i}}{\sum A_{i}}
$$

Onde

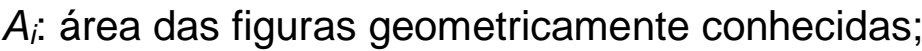

ri: distância do centro de gravidade de cada $A_{i}$ à face mais comprimida.

O momento fletor $(M)$ na seção transversal analisada é o produto da força de reação da região comprimida $\left(R_{c c}\right)$ pela distância $(r)$ do centro de gravidade da área comprimida até a barra de aço tracionada, conforme pode ser visto na Figura 2.

$$
\begin{gathered}
\text { Momento }=\text { Força } \cdot \text { braço de alavanca } \\
M=R_{c c} \cdot r
\end{gathered}
$$

Onde: $r=d-x_{c g}$

Logo temos:

$$
M=0,85 \cdot f_{c d} \cdot A_{c c} \cdot\left(d-x_{c g}\right)
$$

Fazendo as manipulações algébricas da equação acima, chega-se a funções de graus diversos que variam de acordo com a seção transversal da viga. Normalmente, temse o grau 2 para seções retangulares. Uma das raízes dessas funções é a posição da linha neutra no elemento estrutural.

\subsection{LINHA NEUTRA EM VIGA RETANGULAR}

Com base no modelo apresentado no item 2.1 deste artigo, foi obtida a posição da linha neutra para uma viga retangular. A fórmula encontrada para esse tipo de seção transversal pode ser vista em diversos livros, mas o objetivo aqui é demonstrar a dedução da mesma. 
A Figura 3 mostra a posição da linha neutra e o gráfico de tensão do concreto para uma viga de seção transversal retangular. $O$ equilíbrio de momento é feito, dessa forma, tem-se a separação das regiões tracionada e comprimida.

Figura 3 - Linha neutra em viga retangular.

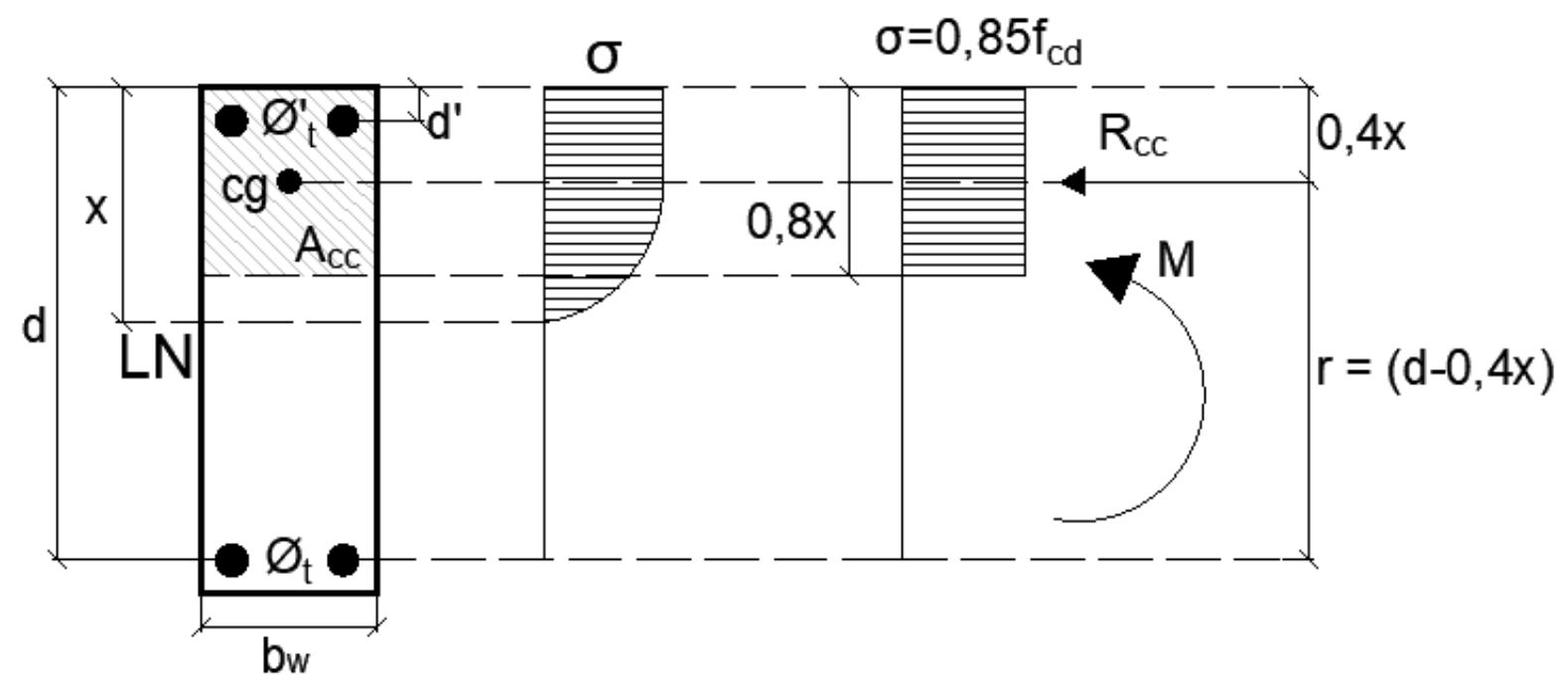

O equilíbrio de momento se dá pelo somatório do momento fletor solicitante e pelo momento gerado pala força de reação da região comprimida de concreto. Com isso, fazendo uso da equação 4 , temos a expressão abaixo.

$$
M=0,85 \cdot f_{c d} \cdot A_{c c} \cdot(d-0,4 x)
$$

A área de concreto da região comprimida é calculada em função do modelo do gráfico de tensão adotado.

$$
A_{c c}=b_{w} \cdot 0,8 x
$$

Fazendo a substituição da equação 6 na equação 5 , temos a expressão final para o obter a posição da linha neutra. 


$$
\begin{gathered}
M=0,85 \cdot f_{c d} \cdot b_{w} \cdot 0,8 x \cdot(d-0,4 x) \\
\frac{M}{0,85 \cdot f_{c d} \cdot b_{w}}=0,8 d x-0,32 x^{2}
\end{gathered}
$$

Note que a equação 7 é do $2^{\circ}$ grau e suas raízes podem ser facilmente encontradas, conforme demonstração a seguir. A maior raiz dessa equação é desprezada, pois, não tem sentido físico para o problema.

$$
\begin{gathered}
x=\frac{0,32 x^{2}-0,8 d x+\frac{M}{0,85 \cdot f_{c d} \cdot b_{w}}=0}{-(-0,8 d)-\sqrt{(-0,8 d)^{2}-4 \cdot 0,32 \cdot\left(\frac{M}{0,85 \cdot f_{c d} \cdot b_{w}}\right)}} \\
x=\frac{0,8 d-\sqrt{0,64 d^{2}-1,28 \cdot\left(\frac{M}{0,85 \cdot f_{c d} \cdot b_{w}}\right)}}{0,64} \\
\left.x=\frac{0,8 d-0,8 d \sqrt{1-\frac{1,28}{0,64 d^{2} \cdot\left(\frac{M}{0,85 \cdot f_{c d} \cdot b_{w}}\right)}}}{0,64}\right) \\
x=1,25 d\left(1-\sqrt{1-\frac{M}{0,425 \cdot d^{\circ} \cdot f c d^{\cdot} \cdot b_{w}}}\right)
\end{gathered}
$$

A posição da linha neutra é calculada com o uso da equação 8, porém, deve-se observar os limites estabelecidos pela NBR 6118 (ABNT, 2014). Esses limites visam garantir a segurança da estrutura.

\subsection{LINHA NEUTRA EM VIGA I E VIGA T SEM MÍSULA}

De modo semelhante às seções retangulares, encontra-se a linha neutra em outros tipos de vigas, aplicando o modelo apresentado no item 2.1 acima. Deve-se observar que as seções I e T não possuem as mesmas dimensões ao longo da altura, devido a isso, devem-se fazer os cálculos para duas hipóteses: linha neutra na mesa e linha neutra na alma. As Figuras 4 e 5 mostram os esquemas de todas as considerações feitas nesse tipo de viga. 
Figura 4 - Linha neutra em viga I sem mísula.

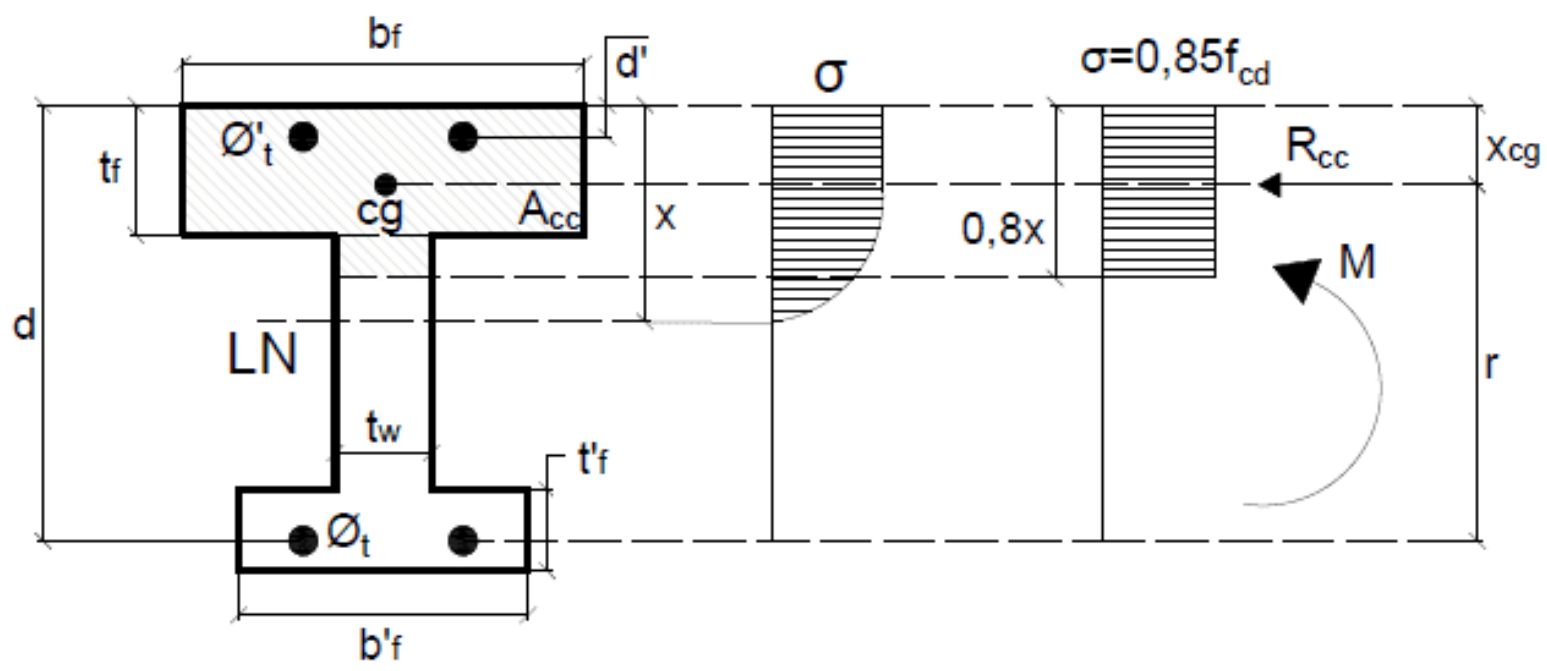

Fonte: própria

Figura 5 - Linha neutra em viga T sem mísula.

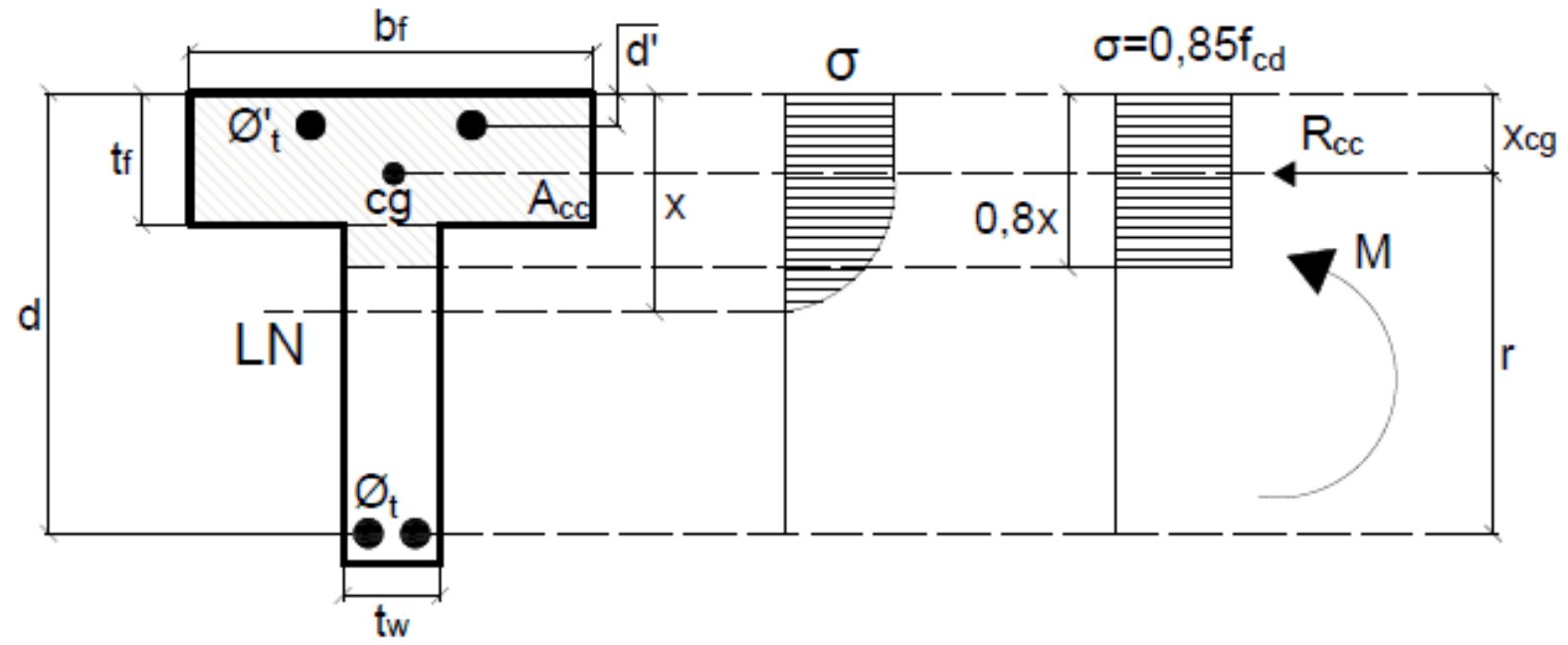

Fonte: própria

Quando a linha neutra passar na mesa da viga, é válida a equação 8, porém, deve se substituir o "bu" pelo "bf", dessa forma temos a equação 9.

$$
x=1,25 d\left(1-\sqrt{1-\frac{M}{0,425 \cdot d^{2} \cdot f_{c d} \cdot b f}}\right)
$$


Quando a linha neutra passar sobre a alma da viga, é feito o mesmo equilíbrio de momento, porém, nessa parte, as mudanças ficam na área de concreto sob compressão $\left(A_{c c}\right)$ e no seu centro de gravidade. Isso ocorre devido à geometria da seção transversal. Usando a equação 3 , encontra-se a distância desse centro de gravidade até a face mais comprimida da viga para esse tipo de seção.

$$
\begin{gathered}
x_{c g}=\frac{\sum A_{i} x_{i}}{\sum A_{i}}=\frac{\left(A_{\text {mesa }} \cdot x_{\text {mesa }}\right)+\left(A_{\text {comprimida da alma }} \cdot x_{\text {área comprimida da alma }}\right)}{\left(A_{\text {mesa }}\right)+\left(A_{\text {comprimida da alma }}\right)} \\
x_{c g}=\frac{\left(b_{f} t_{f} \cdot \frac{t_{f}}{2}\right)+\left(t_{w}\left(0,8 x-t_{f}\right) \cdot\left(t_{f}+\frac{\left(0,8 x-t_{f}\right)}{2}\right)\right)}{\left(b_{f} t_{f}\right)+\left(t_{w}\left(0,8 x-t_{f}\right)\right)} \\
x_{c g}=\frac{\left(0,5 b_{f} t_{f}^{2}\right)+\left(t_{w}\left(0,8 x-t_{f}\right) \cdot\left(0,5 t_{f}+0,4 x\right)\right)}{\left(b_{f} t_{f}\right)+\left(t_{w}\left(0,8 x-t_{f}\right)\right)}
\end{gathered}
$$

A área de concreto comprimida fica conforme a equação 11.

$$
A_{c c}=\left(b_{f} t_{f}\right)+\left(t_{w}\left(0,8 x-t_{f}\right)\right)
$$

Fazendo o equilíbrio de momento, com uso da equação 4, temos a expressão abaixo.

$$
\begin{gathered}
\frac{M}{0,85 \cdot f_{c d}}=A_{c c} \cdot\left(d-x_{c g}\right) \\
\frac{M}{0,85 \cdot f_{c d}}=\left[A_{c c}\right] d-\left[A_{c c}\right] x_{c g} \\
\frac{M}{0,85 \cdot f_{c d}}=\left[\left(b_{f} t_{f}\right)+\left(t_{w}\left(0,8 x-t_{f}\right)\right)\right] d \\
-\left[\left(b_{f} t_{f}\right)+\left(t_{w}\left(0,8 x-t_{f}\right)\right)\right] \cdot\left[\frac{\left(0,5 b_{f} t_{f}{ }^{2}\right)+\left(t_{w}\left(0,8 x-t_{f}\right) \cdot\left(0,5 t_{f}+0,4 x\right)\right)}{\left(b_{f} t_{f}\right)+\left(t_{w}\left(0,8 x-t_{f}\right)\right)}\right] \\
\frac{M}{0,85 \cdot f_{c d}}=d b_{f} t_{f}+d t_{w}\left(0,8 x-t_{f}\right)-\left(0,5 b_{f} t_{f}{ }^{2}\right)-\left(t_{w}\left(0,8 x-t_{f}\right) \cdot\left(0,5 t_{f}+0,4 x\right)\right) \\
\frac{M}{0,85 \cdot f_{c d}}=d b_{f} t_{f}+0,8 d t_{w} x-d t_{w} t_{f}-0,5 b_{f} t_{f}{ }^{2}-0,4 t_{w} t_{f} x+0,5 t_{w} t_{f}{ }^{2}-0,32 t_{w} x^{2}+0,4 t_{w} t_{f} x \\
0,32 t_{w} x^{2}-0,8 d t_{w} x+\frac{M}{0,85 \cdot f c d}-d t_{f}\left(b_{f}-t_{w}\right)+0,5 t_{f}{ }^{2}\left(b_{f}-t_{w}\right)=0
\end{gathered}
$$


Observe que a equação 12 também é do $2^{\circ}$ grau. Da mesma forma das seções retangulares, a maior raiz dessa equação é desprezada, pois, não tem sentido físico para o problema.

$$
\begin{aligned}
& x=\frac{-\left(-0,8 d t_{w}\right)-\sqrt{\left(-0,8 d t_{w}\right)^{2}-4 \cdot 0,32 \cdot\left(\frac{M}{0,85 \cdot f_{c d}}-d t_{f}\left(b_{f}-t_{w}\right)+0,5 t_{f}^{2}\left(b_{f}-t_{w}\right)\right)}}{2 \cdot 0,32 t_{w}} \\
& x=\frac{0,8 d t_{w}-\sqrt{0,64 d^{2} t_{w}{ }^{2}-1,28 t_{w} \cdot\left(\frac{M}{0,85 \cdot f_{c d}}-d t_{f}\left(b_{f}-t_{w}\right)+0,5 t_{f}{ }^{2}\left(b_{f}-t_{w}\right)\right)}}{0,64 t_{w}} \\
& x=\frac{0,8 d t_{w}-0,8 d t_{w} \sqrt{1-\frac{1,28 t_{w}}{0,64 d^{2} t_{w}^{2}} \cdot\left(\frac{M}{0,85 \cdot f_{c d}}-d t_{f}\left(b_{f}-t_{w}\right)+0,5 t_{f}^{2}\left(b_{f}-t_{w}\right)\right)}}{0,64 t_{w}} \\
& x=1,25 d\left(1-\sqrt{1-\frac{2}{d^{2} t_{w}} \cdot\left(\frac{M}{0,85 \cdot f_{c d}}-d t_{f}\left(b_{f}-t_{w}\right)+0,5 t_{f}^{2}\left(b_{f}-t_{w}\right)\right)}\right)
\end{aligned}
$$

Para as vigas do tipo T e I, a posição da linha neutra (LN) é calculada com o uso das equações 9 e 13, respectivamente, para LN na mesa e LN na alma. Deve-se observar a espessura da mesa para determinar o limite de transição entre a mesa e a alma da viga. Devido aos limites estabelecidos pela NBR 6118 (ABNT, 2014), não se faz necessário o cálculo da linha neutra na mesa inferior da viga I.

\subsection{LINHA NEUTRA EM VIGA I E EM VIGA T COM MÍSULA NA MESA}

Nas estruturas correntes, é comum o uso de mísula nas vigas do tipo T e I. Esse tipo de viga pode ser calculado, de forma aproximada, conforme o item anterior. Mas, para encontrar resultados mais fidedignos, será apresentado aqui uma maneira de obter a posição da linha neutra vigas T e I com mísula.

As Figuras 6 e 7 exemplificam, de maneira objetiva, a forma da seção transversal e seus gráficos de tensões aproximados para esse tipo de viga. 
Figura 6 - Linha neutra em viga I com mísula na mesa.

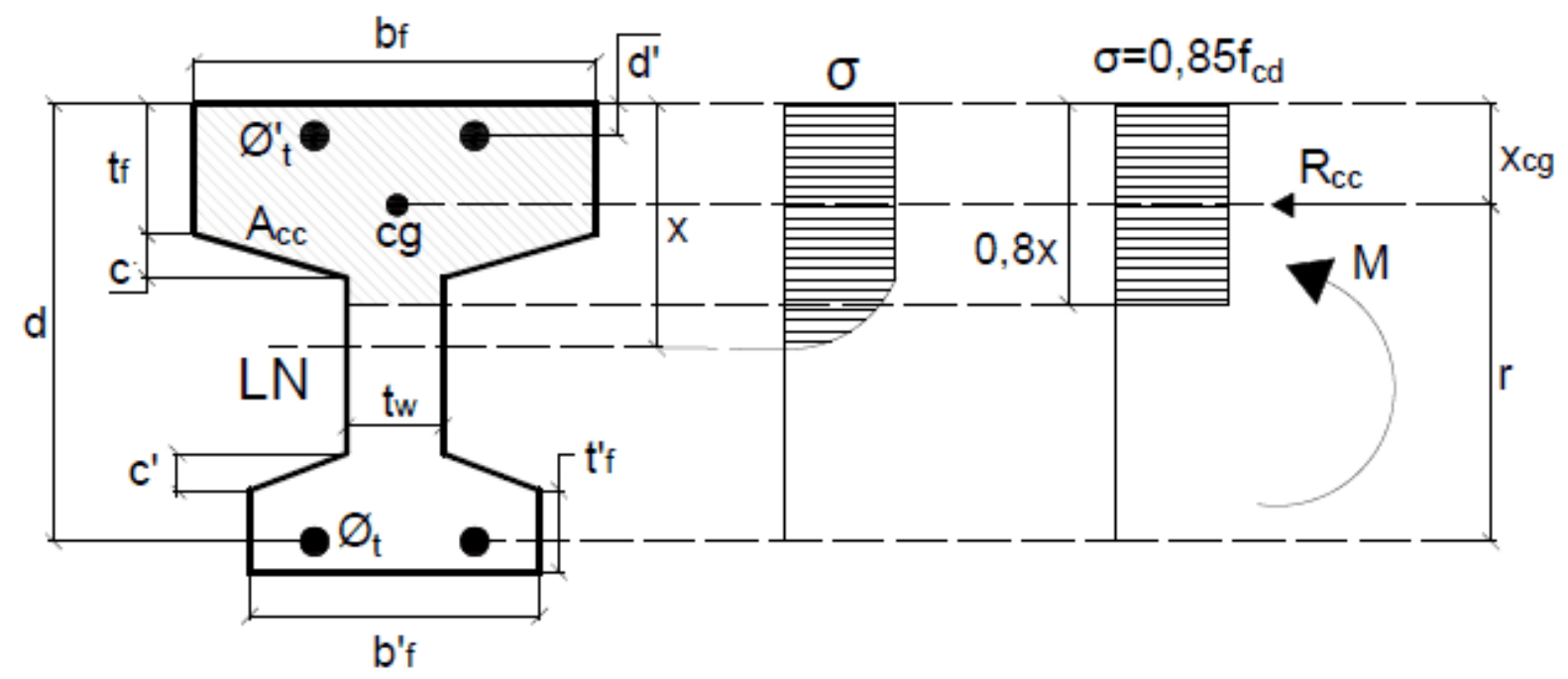

Fonte: própria

Figura 7 - Linha neutra em viga T com mísula na mesa.

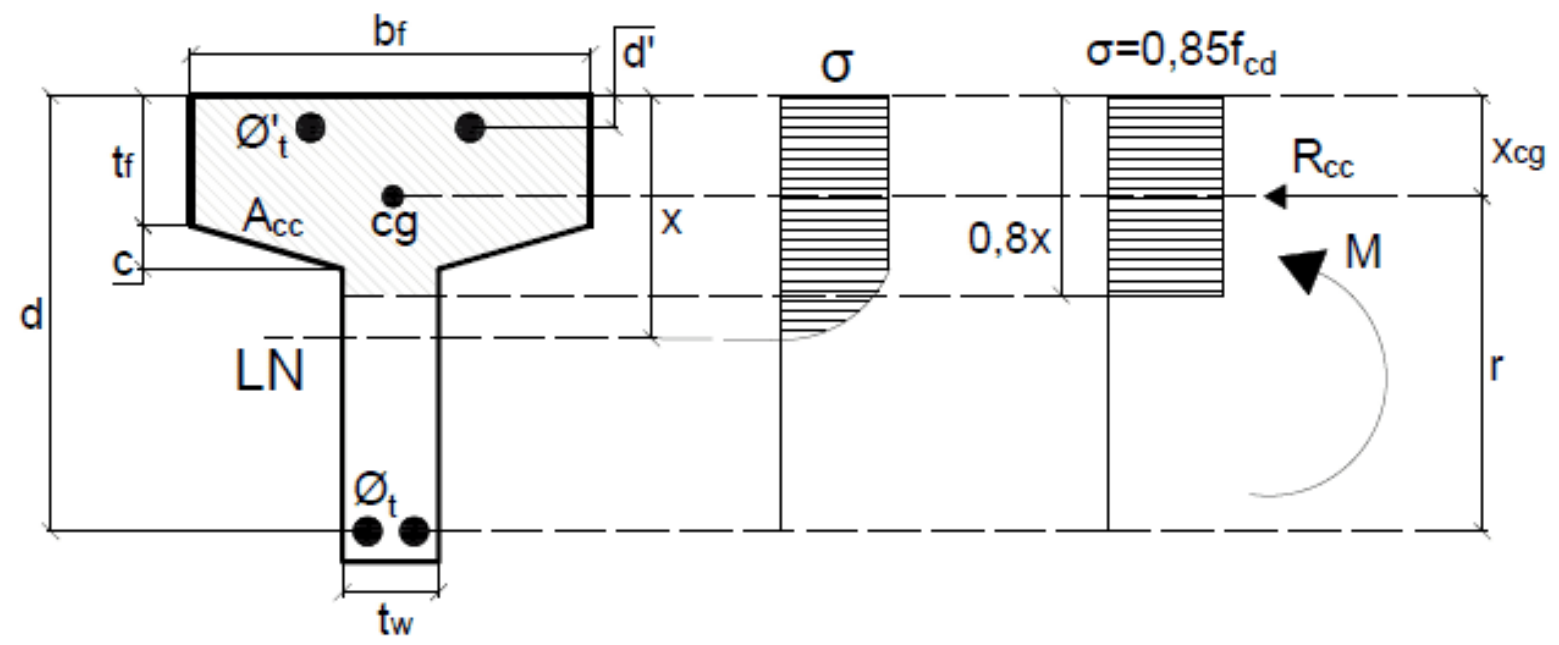

Fonte: própria

Nesse tipo de viga, há três hipóteses. A primeira é o caso da linha neutra passar na mesa da viga, com isso, é válida a equação 9 . A segunda ocorre quando a linha neutra passa sobre a região com mísula. A terceira é o caso da linha neutra na alma da viga, nesse caso, teremos uma solução semelhante à equação 13 , porém, com alguns ajustes em função da área de concreto sob compressão $\left(A_{c c}\right)$. Como a primeira hipótese já possui uma equação definida, iniciaremos neste item a dedução para a 
segunda hipótese. Com a aplicação da equação 3 , encontra-se a distância do centroide da região sob compressão até a face mais comprimida da viga, conforme Figura 8.

Figura 8 - Centro de gravidade da área comprimida.

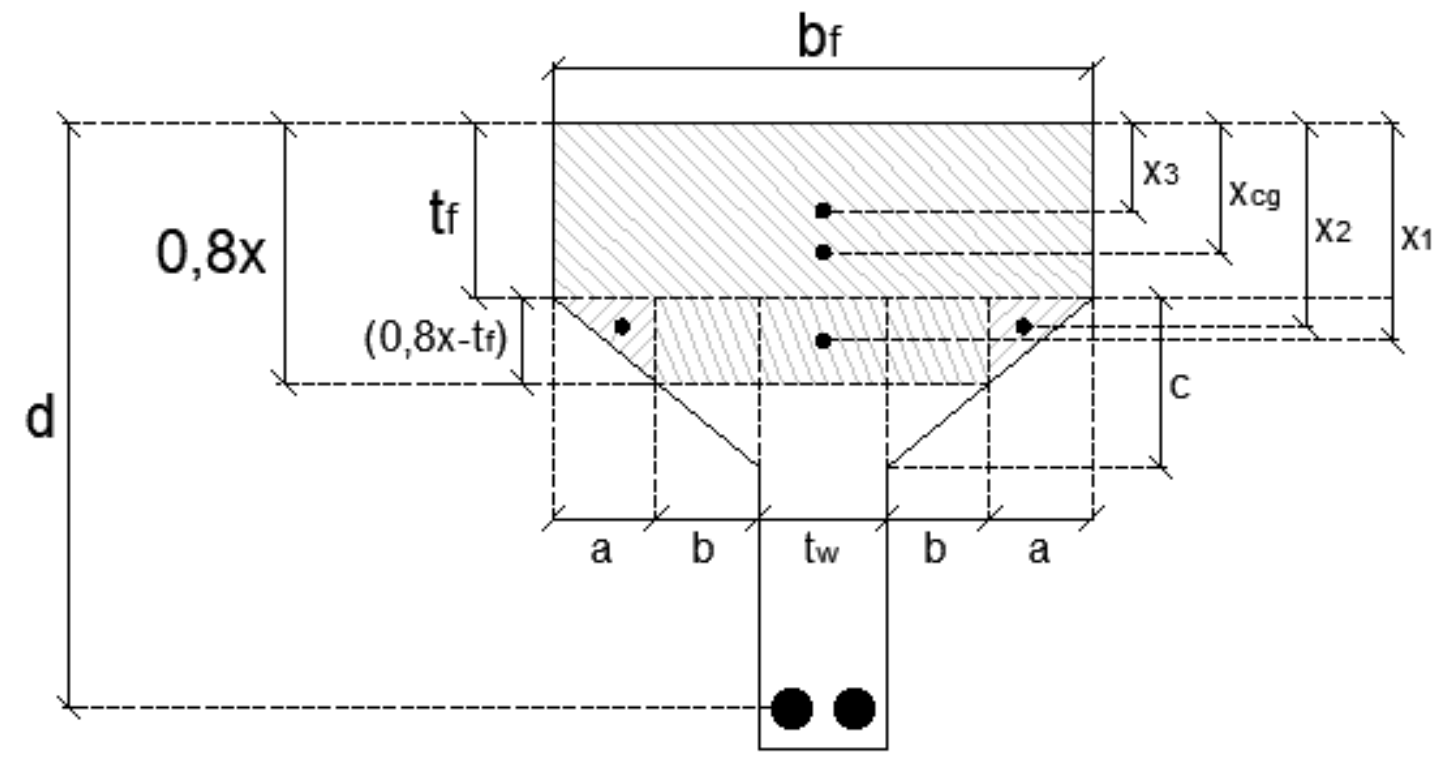

Fonte: própria

Nesse caso, onde há regiões triangulares na seção transversal, é mais complicada a dedução das equações. Devido a isso, iniciaremos com os cálculos das distâncias $x_{1}$, $x_{2}$ e $x_{3}$ com suas respectivas áreas geométricas.

O valor de $x_{1}$ é a distância do centro de gravidade do retângulo da região com mísula até a face superior da viga, conforme a Figura 8.

$$
x_{1}=t_{f}+\frac{0,8 x-t_{f}}{2} \rightarrow x_{1}=\frac{0,8 x+t_{f}}{2}
$$

O valor de $x_{2}$ é a distância do centro de gravidade dos triângulos à face superior da viga, conforme a Figura 8.

$$
x_{2}=t_{f}+\frac{0,8 x-t_{f}}{3} \rightarrow x_{2}=\frac{0,8 x+2 t_{f}}{3}
$$


O valor de $x_{3}$ é a distância do centro de gravidade do retângulo, referente à mesa, até a face superior da viga, conforme a Figura 8.

$$
x_{3}=\frac{t_{f}}{2}
$$

Cálculo da área $1\left(A_{1}\right)$ referente ao retângulo de $x_{1}$.

$$
A_{1}=\left(2 b+t_{w}\right) \cdot\left(0,8 x-t_{f}\right)
$$

Onde, por semelhança de triângulo, "b" é igual a:

$$
\begin{aligned}
& b=0,5\left(b_{f}-t_{w}\right)-\frac{0,4}{c}\left(b_{f}-t_{w}\right) x+\frac{0,5}{c}\left(b_{f}-t_{w}\right) t_{f} \\
& \therefore A_{1}=\frac{-0,64}{c}\left(b_{f}-t_{w}\right) x^{2}+\left[\frac{1,6 t_{f}}{c}\left(b_{f}-t_{w}\right)+0,8 b_{f}\right] x-\left[b_{f}+\frac{t_{f}}{c}\left(b_{f}-t_{w}\right)\right] t_{f}
\end{aligned}
$$

Cálculo da área $2\left(A_{2}\right)$ referente aos dois triângulos de $x_{2}$.

$$
A_{2}=a \cdot\left(0,8 x-t_{f}\right)
$$

Onde, por semelhança de triângulo, "a" é igual a:

$$
\begin{gathered}
a=\frac{0,4}{c}\left(b_{f}-t_{w}\right) x-\frac{0,5}{c}\left(b_{f}-t_{w}\right) t_{f} \\
\therefore A_{2}=\frac{0,32}{c}\left(b_{f}-t_{w}\right) x^{2}-\frac{0,8 t_{f}}{c}\left(b_{f}-t_{w}\right) x+\frac{0,5 t_{f}^{2}}{c}\left(b_{f}-t_{w}\right)
\end{gathered}
$$

Cálculo da área $3\left(A_{3}\right)$ referente ao retângulo de $x_{3}$.

$$
A_{3}=b_{f} \cdot t_{f}
$$


Com as equações de $A_{1}, A_{2}, A_{3}, x_{1}, x_{2}$ e $x_{3}$, é possível calcular o centro de gravidade da área de concreto comprimida, substituindo-as na equação 3 .

$$
x_{c g}=\frac{\sum A_{i} x_{i}}{\sum A_{i}} \rightarrow x_{c g}=\frac{A_{1} x_{1}+A_{2} x_{2}+A_{3} x_{8}}{A_{1}+A_{2}+A_{8}}
$$

Fazendo o equilíbrio de momento, com uso da equação 4, temos a expressão abaixo.

$$
\begin{gathered}
M=0,85 \cdot f_{c d} \cdot\left(A_{1}+A_{2}+A_{3}\right) \cdot\left(d-\frac{A_{1} x_{1}+A_{2} x_{2}+A_{3} x_{3}}{A_{1}+A_{2}+A_{3}}\right) \\
\frac{M}{0,85 \cdot f_{c d}}=\left\{A_{1}+A_{2}+A_{3}\right\} d-\left[A_{1} x_{1}\right]-\left[A_{2} x_{2}\right]-\left[A_{3} x_{3}\right] \\
\frac{M}{0,85 \cdot f_{c d}}=\left\{\frac{-0,64}{c}\left(b_{f}-t_{w}\right) x^{2}+\left[\frac{1,6 t_{f}}{c}\left(b_{f}-t_{w}\right)+0,8 b_{f}\right] x-\left[b_{f}+\frac{t_{f}}{c}\left(b_{f}-t_{w}\right)\right] t_{f}+\right. \\
\left.\frac{0,32}{c}\left(b_{f}-t_{w}\right) x^{2}-\frac{0,8 t_{f}}{c}\left(b_{f}-t_{w}\right) x+\frac{0,5 t_{f}^{2}}{c}\left(b_{f}-t_{w}\right)+b_{f} \cdot t_{f}\right\} d- \\
{\left[\left(\frac{-0,64}{c}\left(b_{f}-t_{w}\right) x^{2}+\left[\frac{1,6 t_{f}}{c}\left(b_{f}-t_{w}\right)+0,8 b_{f}\right] x-\left[b_{f}+\frac{t_{f}}{c}\left(b_{f}-t_{w}\right)\right] t_{f}\right) \cdot\left(\frac{0,8 x+t_{f}}{2}\right)\right]-} \\
{\left[\left(\frac{0,32}{c}\left(b_{f}-t_{w}\right) x^{2}-\frac{0,8 t_{f}}{c}\left(b_{f}-t_{w}\right) x+\frac{0,5 t_{f}^{2}}{c}\left(b_{f}-t_{w}\right)\right) \cdot\left(\frac{0,8 x+2 t_{f}}{3}\right)\right]-\left[\left(b_{f} \cdot t_{f}\right) \cdot\left(\frac{t_{f}}{2}\right)\right]} \\
{\left[\frac{0,512\left(b_{f}-t_{w}\right)}{3 c}\right] x^{3}-\left[\frac{0,32 d\left(b_{f}-t_{w}\right)}{c}+\frac{0,32 t_{f}\left(b_{f}-t_{w}\right)}{c}+0,32 b_{f}\right] x^{2}+} \\
{\left[\frac{0,8 t_{f} d\left(b_{f}-t_{w}\right)}{c}+0,8 d b_{f}\right] x-\left[\frac{0,5 t_{f}{ }^{2} d\left(b_{f}-t_{w}\right)}{c}-\frac{0,5 t_{f}^{3}\left(b_{f}-t_{w}\right)}{3 c}+\frac{M}{0,85 \cdot f_{c d}}\right]=0}
\end{gathered}
$$

Com a equação 21, pode-se calcular a posição da linha neutra na região com mísula. Note que essa equação é do 3o grau, com isso, se encontra três raízes. Uma dessas raízes, normalmente a menor, é a posição da linha neutra, as outras duas não terão sentido físico, por isso, devem ser desconsideradas.

Seguindo o mesmo procedimento, encontra-se a posição da $L N$ para a terceira hipótese. Aplicando a equação 3 , podemos encontrar a distância do centro de gravidade da área sob compressão até a face mais comprimida da viga. As medidas de cada dimensão, para aplicação das equações pertinentes, estão dispostas da Figura 9. 
Figura 9 - Centro de gravidade da área comprimida.

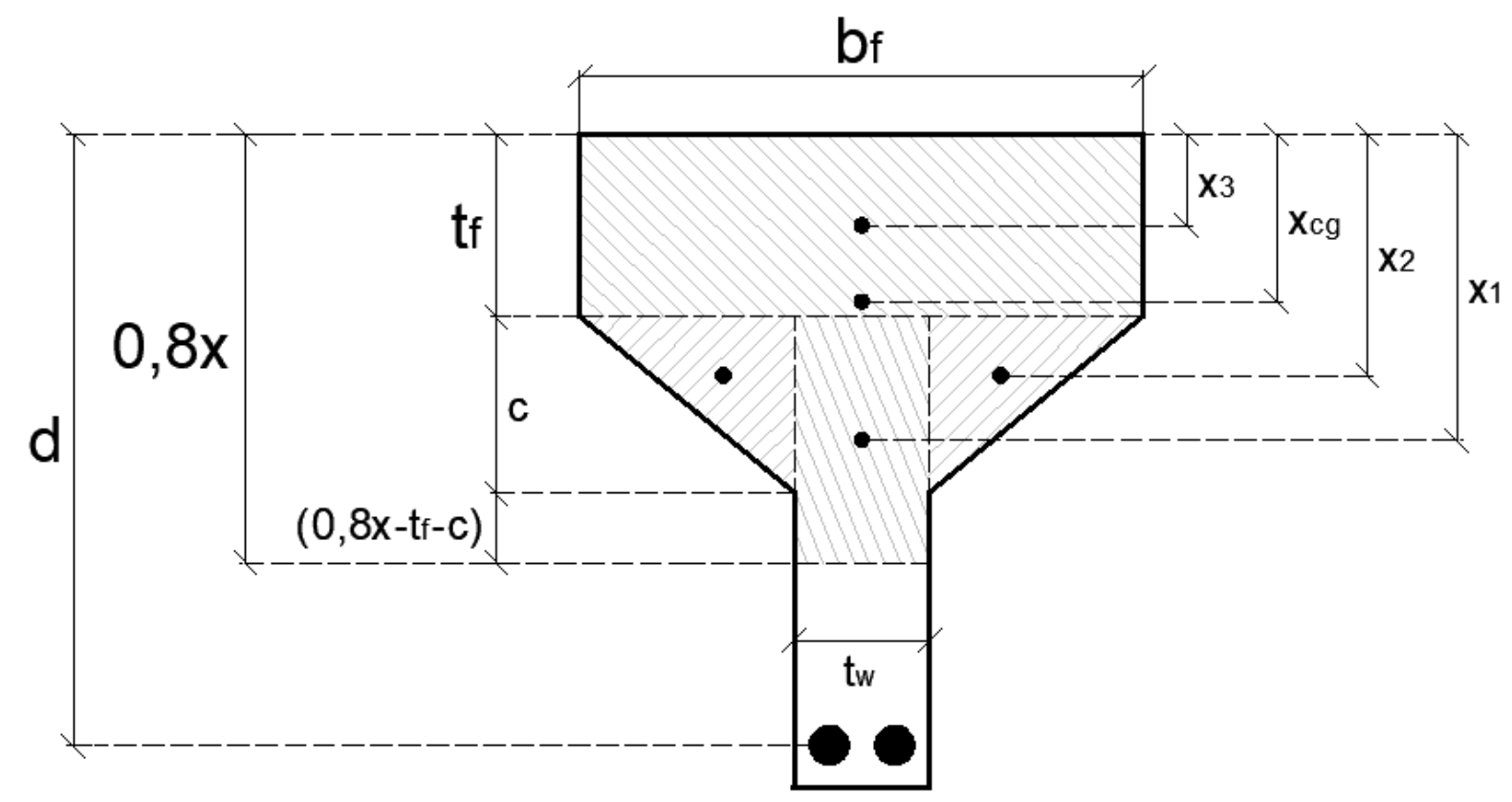

Fonte: própria

A fim de facilitar as manipulações algébricas, iniciou-se aqui a obtenção das distâncias $x_{1}, x_{2}$ e $x_{3}$ e suas respectivas áreas geométricas. $O$ valor de $x_{1}$ é a distância do centro de gravidade do retângulo com hachura na alma até a face superior da viga.

$$
x_{1}=t_{f}+\frac{0,8 x-t_{f}}{2} \rightarrow x_{1}=0,4 x+0,5 t_{f}
$$

O valor de $x_{2}$ é a distância do centro de gravidade dos triângulos até a face superior da viga, conforme visto na Figura 9.

$$
x_{2}=t_{f}+\frac{c}{3}
$$

O valor de $x_{3}$ é a distância do centro de gravidade do retângulo, referente à mesa, até a face superior da viga.

$$
x_{3}=\frac{t_{f}}{2}
$$


Cálculo da área $1\left(A_{1}\right)$ referente ao retângulo de $x_{1}$.

$$
A_{1}=t_{w} \cdot\left(0,8 x-t_{f}\right) \rightarrow A_{1}=0,8 t_{w} x-t_{w} t_{f}
$$

Cálculo da área $2\left(\mathrm{~A}_{2}\right)$ referente aos dois triângulos de $\mathrm{x}_{2}$.

$$
A_{2}=c \cdot\left(\frac{b_{f}-\tau_{w}}{2}\right) \rightarrow A_{2}=0,5 c b_{f}-0,5 c t_{w}
$$

Cálculo da área $3\left(A_{3}\right)$ referente ao retângulo de $x_{3}$.

$$
A_{3}=b_{f} \cdot t_{f}
$$

Cálculo do centro de gravidade da área de concreto comprimida à face superior da viga.

$$
x_{c g}=\frac{\sum A_{i} x_{i}}{\sum A_{i}} \rightarrow x_{c g}=\frac{A_{1} x_{1}+A_{2} x_{2}+A_{8} x_{8}}{A_{1}+A_{2}+A_{8}}
$$

Com o equilíbrio de momento na seção transversal da viga, usando a equação 4 , temos a expressão abaixo.

$$
\begin{gathered}
M=0,85 \cdot f_{c d} \cdot\left(A_{1}+A_{2}+A_{3}\right) \cdot\left(d-\frac{A_{1} x_{1}+A_{2} x_{2}+A_{3} x_{3}}{A_{1}+A_{2}+A_{3}}\right) \\
\frac{M}{0,85 \cdot f_{c d}}=\left\{A_{1}+A_{2}+A_{3}\right\} d-\left[A_{1} x_{1}\right]-\left[A_{2} x_{2}\right]-\left[A_{3} x_{3}\right] \\
\frac{M}{0,85 \cdot f_{c d}}=\left\{0,8 t_{w} x-t_{w} t_{f}+0,5 c b_{f}-0,5 c t_{w}+b_{f} \cdot t_{f}\right\} d \\
-\left[\left(0,8 t_{w} x-t_{w} t_{f}\right) \cdot\left(0,4 x+0,5 t_{f}\right)\right]-\left[\left(0,5 c b_{f}-0,5 c t_{w}\right) \cdot\left(t_{f}+\frac{c}{3}\right)\right] \\
-\left[\left(b_{f} \cdot t_{f}\right) \cdot\left(0,5 t_{f}\right)\right] \\
\therefore\left[0,32 t_{w}\right] x^{2}-\left[0,8 d t_{w}\right] x-\left[\left(0,5 t_{f}-d-0,5 c\right)\left(t_{w} t_{f}-b_{f} t_{f}\right)+\right. \\
+\left(0,5 c d-\frac{0,5 c^{2}}{3}\right)\left(b_{f}-t_{w}\right)-\frac{M}{0,85 \cdot f c d}=0
\end{gathered}
$$


Resolvendo a equação 29 , encontram-se suas duas raízes. A menor delas é a posição da linha neutra na viga. A maior raiz deve ser desconsiderada para esse problema.

$$
\begin{gathered}
{\left[0,32 t_{w}\right] x^{2}-\left[0,8 d t_{w}\right] x+C=0} \\
C=-\left[\left(0,5 t_{f}-d-0,5 c\right)\left(t_{w} t_{f}-b_{f} t_{f}\right)+\left(0,5 c d-\frac{0,5 c^{2}}{3}\right)\left(b_{f}-t_{w}\right)-\frac{M}{0,85 \cdot f_{c d}}\right] \\
x=\frac{-\left(-0,8 d t_{w}\right)-\sqrt{\left(0,8 d t_{w}\right)^{2}-4\left(0,32 t_{w}\right) C}}{2\left(0,32 t_{w}\right)} \\
x=1,25 d\left(1-\sqrt{1-\frac{2 c}{d^{2} t_{w}}}\right)
\end{gathered}
$$

Para essa forma de viga, tipo T e I com mísula, a posição da linha neutra (LN) é obtida pelas equações 9,21 e 30 , respectivamente, para $L N$ na mesa, $L N$ na região com mísula e LN na alma. Da mesma forma que foi exposto no item 2.3, deve-se observar os limites estabelecidos pela NBR 6118 (ABNT, 2014).

\subsection{LINHA NEUTRA EM VIGA TRAPEZOIDAL}

Esse é um tipo de viga pouco usado, mas há algumas aplicações de acordo com a necessidade do projeto. Como pôde ser observado ao longo deste estudo, a obtenção da posição da linha neutra é feita pelo equilíbrio de momento na seção transversal. $O$ modelo apresentado aqui está conforme a Figura 10, ou seja, não é válido para base maior na parte inferior.

Figura 10 - Linha neutra em viga trapezoidal.

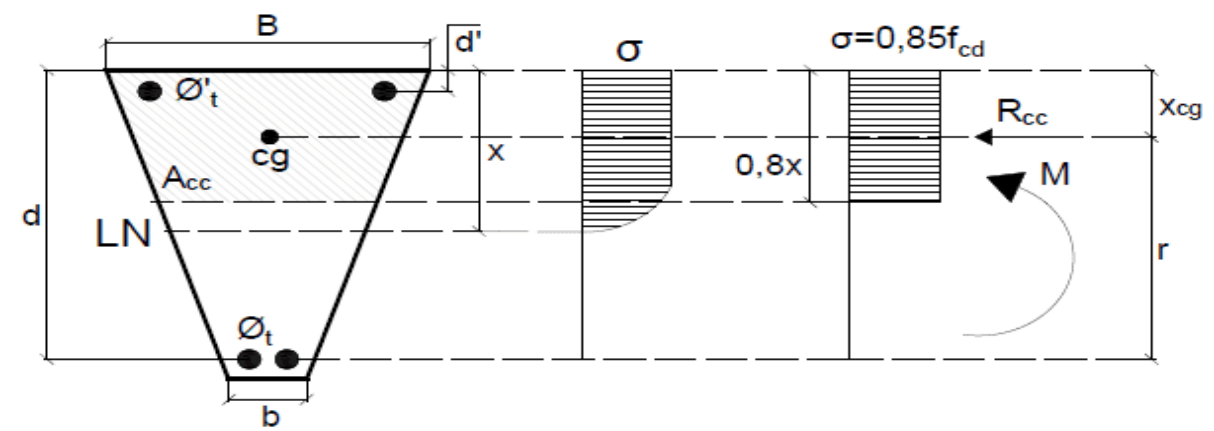

Fonte: própria

RC: 39830

Disponível em: https://www.nucleodoconhecimento.com.br/engenharia-civil/calculo-da-linha 
Figura 11 - Centro de gravidade da área comprimida.

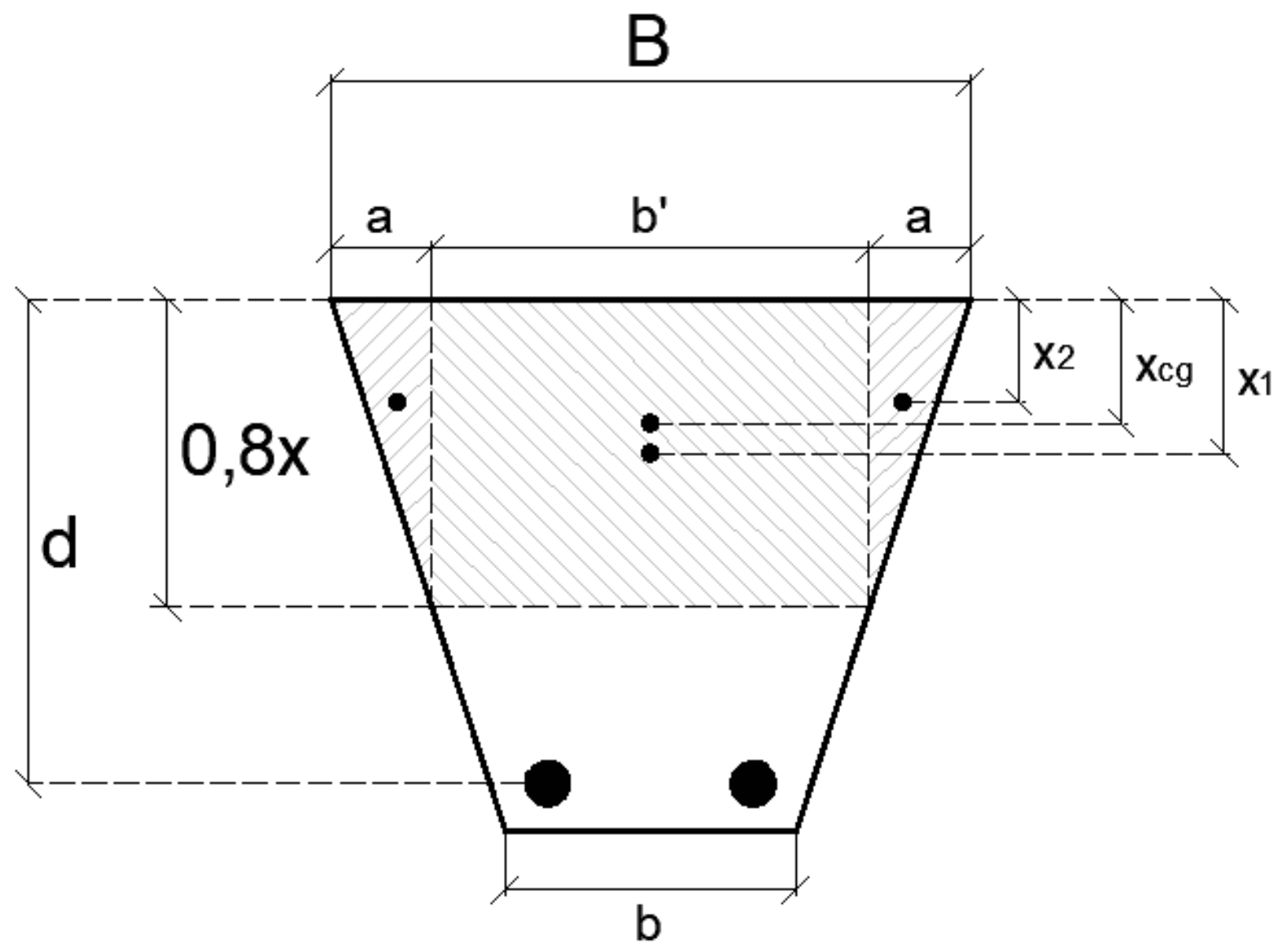

Fonte: própria

Para esse tipo de viga, em que há regiões triangulares na seção transversal, a equação para determinar a linha neutra é semelhante à viga $T$ e I com mísula. $O$ cálculo é iniciado com as distâncias $x_{1}$ e $x_{2}$ com suas respectivas áreas geométricas.

O valor de $x_{1}$ é a distância do centro de gravidade do retângulo com hachura à face superior da viga, conforme indicado na Figura 11.

$$
x_{1}=\frac{0,8 x}{2} \rightarrow x_{1}=0,4 x
$$

O valor de $x_{2}$ é a distância do centro de gravidade dos triângulos à face superior da viga, conforme indicado na Figura 11. 


$$
x_{2}=\frac{0,8 x}{3}
$$

Cálculo da área $1\left(A_{1}\right)$ referente ao retângulo de $x_{1}$.

$$
A_{1}=0,8 x \cdot b^{\prime}
$$

Onde, por semelhança de triângulo e de forma aproximada, b’é igual a:

$$
\begin{gathered}
b^{\prime}=B-\frac{0,8 x}{d}(B-b) \\
\therefore A_{1}=-\frac{0,64}{d}(B-b) x^{2}+0,8 B x
\end{gathered}
$$

Cálculo da área $2\left(A_{2}\right)$ referente aos dois triângulos de $x_{2}$.

$$
A_{2}=a \cdot 0,8 x
$$

Onde, por semelhança de triângulo e de forma aproximada, "a" é igual a:

$$
\begin{aligned}
a & =\frac{0,4}{d}(B-b) x \\
\therefore A_{2} & =\frac{0,32}{d}(B-b) x^{2}
\end{aligned}
$$

Com as equações das áreas e as distâncias $x_{i}$ acima calculadas, aplicando a equação 3, tem-se o centro de gravidade da área de concreto comprimida.

$$
x_{c g}=\frac{\sum A_{i} x_{i}}{\sum A_{i}} \rightarrow x_{c g}=\frac{A_{1} x_{1}+A_{2} x_{2}}{A_{1}+A_{2}}
$$

Do mesmo modo das vigas anteriores, fazendo o equilíbrio de momento com a equação 4, temos a seguinte expressão. 


$$
\begin{gathered}
M=0,85 \cdot f_{c d} \cdot\left(A_{1}+A_{2}\right) \cdot\left(d-\frac{A_{1} x_{1}+A_{2} x_{2}}{A_{1}+A_{2}}\right) \\
\frac{M}{0,85 \cdot f_{c d}}=\left\{A_{1}+A_{2}\right\} d-\left[A_{1} x_{1}\right]-\left[A_{2} x_{2}\right] \\
\frac{M}{0,85 \cdot f_{c d}}=\left\{-\frac{0,64}{d}(B-b) x^{2}+0,8 B x+\frac{0,32}{d}(B-b) x^{2}\right\} d- \\
{\left[\left(-\frac{0,64}{d}(B-b) x^{2}+0,8 B x\right) \cdot(0,4 x)\right]-} \\
{\left[\left(\frac{0,32}{d}(B-b) x^{2}\right) \cdot\left(\frac{0,8 x}{3}\right)\right]} \\
\therefore\left[\frac{0,512}{3 d}(B-b)\right] x^{3}-[0,64 B-0,32 b] x^{2}+[0,8 d B] x-\frac{M}{0,85 \cdot f_{c d}}=0
\end{gathered}
$$

Observe que a equação 36 é do $3^{\circ}$ grau, com isso, ao resolvê-la, encontra-se três raízes. Normalmente, a menor raiz é a solução do problema. Esse tipo de equação não possui uma fórmula para resolvê-la, então, a melhor maneira é usar algum software. Há métodos interativos para solucionar polinômio com grau maior que dois. Um desses métodos é o de Gauss-Seidel apresentado por Boldrini et al (1980).

\section{CONCLUSÃO}

Mediante as analises feitas, ficou esclarecida a forma como é calculada a posição da linha neutra em vigas submetidas à flexão. Para outros tipos de seções transversais em vigas não abordadas aqui, pode-se seguir o mesmo modelo de cálculo demonstrado ao longo desse estudo. Ficou notório que quando a seção transversal é composta de figuras retangulares, chega-se a uma equação do $2^{\circ}$ grau, já as que possuem alguma parte triangular, o problema é resolvido com equações do $3^{\circ}$ grau. Para essas equações do $3^{\circ}$ grau, é necessário adotar algum método matemático para encontrar as raízes, por exemplo, métodos iterativos, pois não há fórmulas para resolvê-las. 


\section{REFERÊNCIAS}

ASSOCIAÇÃO BRASILEIRA DE NORMAS TÉCNICAS. NBR 6118: projeto de estruturas de concreto: procedimento. Rio de Janeiro, 2014.

BOLDRINI, J. L. et al. Álgebra Linear. 3. ed. São Paulo: Harper \& Row do Brasil, 1980.

CARVALHO, R. C.; FILHO, J. R. F. Cálculo e Detalhamento de Estruturas Usuais de Concreto Armado. 3. ed. São Carlos: EdUFSCar, 2007.

Enviado: Agosto, 2019.

Aprovado: Outubro, 2019. 